\title{
REASONING ABOUT ACTIONS, KNOWLEDGE AND NORMATIVE ABILITY
}

\author{
XIANWEI LAI ${ }^{1,3}$, SHANLI HU ${ }^{1,2}$, ZHENGYUAN NING ${ }^{3, *}$ \\ I Dept. of Computer Science and Technology, Fuzhou University, Fuzhou 350002, China \\ xianweilai@163.com \\ ${ }^{2}$ Laboratory of Computer Science, Chinese Academy of Sciences, Beijing 100080. China \\ husi@fzu.edu.cn \\ 3 College of Computer and Information, Fujian Agriculture and Forestry University, \\ Fuzhou 350002, China \\ ningzhy@126.com \\ * The Contact Author
}

Abstract: The past five years have witnessed an explosion of interest in the use of cooperation logics for reasoning about multi-agent systems. Since the development of ATL, there are many multi-agent cooperation logics developed as an extension to ATL. The cooperation logic called the Normative Alternating-time Temporal Epistemic Logic (NATEL) is developed to extend ATL. Four key contributions have been made. Firstly, the strong and unrealistic assumption of the other two extended cooperation logics of ATL (ATEL, NATL ${ }^{*}$ ) that different agents are not allowed to control the same actions have been done away with. Secondly, functions that involved actions are given in more detail, so that the relations between actions and knowledge, actions and agents, actions and states can be researched in depth and separately. Thirdly, actions, knowledge and normative ability can be represented in the object language other than only in the underlying semantics. Lastly, since actions, knowledge and normative ability are taken into account at the same time, the expressive power and flexibility of NATEL are much richer than the other two extended cooperation logics of ATL

Key words: ATL, multi-agent systems, cooperation logic, knowledge, actions 


\section{INTRODUCTION}

The past five years have witnessed an explosion of interest in the use of cooperation logics for reasoning about multi-agent systems. There are three main cooperation logics developed: first, the Alternating-time Temporal Logic (ATL) [6], [7], where $\langle\langle G\rangle\rangle$ is used as a cooperation modality (parameterized path quantifier), and formula $<<G>>\varphi$ expressing that agents in coalition $G$ can cooperate to ensure that $\varphi$ holds, that is coalition $G$ have a winning strategy for $\varphi$; second, Coalition Logic (CL) [8], [9] where formula $[G] \varphi$ expressing that coalition $G$ is able to achieve in one move an outcome where $\varphi$ is true, and formula $\left[G^{*}\right] \varphi$ asserting that coalition $G$ can achieve $\varphi$ at some point in the future; third, the Coalition Logic for Propositional Control (CL-PC) [10], where by controlling the propositional atoms, an agent or any coalition he is in can determine what it will achieve. Among these cooperation logics developed, ATL which replaces path quantifiers of CTL by cooperation modalities has received particular attention. Over the last three years, cooperation logics like ATEL (which takes knowledge into account) [11], [12], [13] and NATL* (which takes normative ability into account) [14] have been developed to extend ATL in different ways. They can express properties like additional constrains on actions and common knowledge that ATL can not.

There are problems unresolved in these cooperation logics. Firstly, it is a common approach in these extended cooperation logics but obviously too strong and unpractical an assumption that different agents are not allowed to control the same actions. That is, each agent is associated with a set of actions that he can execute, and it is assumed that these sets of actions are pairwise disjoint because they are owned by different agents. Now let us consider an example where the coalition of agents is a family, surely, members in this coalition share a common action 'turn on the TV' when they have had supper. So, it is obvious that a coalition logic which allows different agents to control the same actions needs to be developed. And this leads to the first effort our paper makes.

Secondly, the ability of agents to cooperate to execute actions (what actions an agent or a coalition of agents can perform) and how this relates to their ability to reach certain states of affairs (the effects of actions) are worth investigated separately, but neither ATL nor its extended cooperation logics (ATEL, NATL*) has adopted such an approach. Regarding this, paper [15] provides two logic modules to achieve the separate investigating effect. Although this approach seems clear and the soundness and completeness of the axiom systems of both of the two logic modules are easier to gained and proved, it seems the two abilities mentioned above can be investigated in more detail within a unified model. 
Lastly, to make good use of these ideas of extension and combine these extended cooperation logics will form a unified cooperation logic whose expressive power and flexibility are much richer than its ancestors. Modalities of knowledge (comes from epistemic logic) and function of normative ability (to deal with real problems, there are additional constraints on the actions that may be performed in any given state) are investigated together. Since unification creates links between these two approaches which had not been connected before, it is desirable to explore the relations between them even if no old questions can be answered and no new questions arise.

\section{UNIFIED MODEL}

Compositions of multi-agent systems can be modeled by the following unified model where a state transition results from choices made by the system components and the environment. This unified model (namely, Action-based Normative Alternating Epistemic Transition Systems) is a straightforward extension of the Action-based Alternating Transition Systems (AATSs) used by Wiebe van der Hoek [13]. It combines the main components of [13], [14], [15], and at the same time contains components of our own. It is the semantic structures to our cooperation logic (NATEL).

Definition 1. (UNIFIED MODEL)

A unified model for the semantics to NATEL is a $(n+12)$-tuple

$M=<S, A g, P, A t, \sim_{1}, \ldots, \sim_{\mathrm{n}}, T_{a l}, T_{c t}, T_{t a}, T_{t c}, T_{v i}, T_{l i s}, \tau, \pi>$, with the following components:

- $S$ is a finite, non-empty set of states;

- $A g=\{1, \ldots, \mathrm{n}\}$ is a finite, non-empty set of agents;

- $P$ is a finite, non-empty set of atomic propositions;

- $A t=A t_{1} \cup \ldots \cup A_{n}$ is the finite, non-empty set of all actions, where each agent $\mathrm{i} \in \mathrm{Ag}$ is associated with a finite, non-empty set $\mathrm{At}_{\mathrm{i}}$ of possible actions; different agents can perform the same action, i.e., for each i, $\mathrm{j} \in \mathrm{Ag}$, and $\mathrm{i} \neq \mathrm{j}$, it may be true that $A t_{1} \cap A t_{j} \neq \phi ;$ then we define an action tuple as $\alpha=<t_{1}, \ldots, t_{k}>$, where $\mathrm{t}_{\mathrm{i}}$ is the action chosen by agent $\mathrm{i}, 1 \leq k \leq n$, and for any agent $i$, she can choose nothing ( $t_{i}$ is null) or choose a common action $\left(i \neq j\right.$, but $\left.t_{i}=t_{j}\right)$; so that the assumption that different agents can not execute the same actions which obviously does not fit human commonsense understanding of the world has been done away with.

- $\sim_{i} \subseteq S \times S$ is an epistemic accessibility relation for each agent $i \in A g$. Each $\sim$ must be an equivalence relation; 
- $T_{a l}: A g \rightarrow A t$ is a function that assigns to each agent $i \in A g$ one action from $A t$. Under this definition, it is assumed that the action assigned to the agent is controlled by this agent;

- $T_{c l}: 2^{A g} \rightarrow 2^{A t}$ is a function that assigns to each coalition $G \subseteq A g$ a subset of actions from At. Under this definition, it is assumed that the subset of actions assigned to the coalition of agents are controlled by this coalition;

- $T_{i a}: A t \rightarrow A g$ is a function that assigns to each action $t \in A t$ one agent from $A g$. It provides us with convenience to find the agent who is exactly controlling this action.

- $T_{l c}: A t \rightarrow 2^{A s}$ is a function that assigns to each action $t \in A t$ a subset of agents from $\mathrm{Ag}$. It provides us with convenience to find the coalition of agents who is exactly controlling this action;

$-T_{s t}: S \rightarrow 2^{A t}$ is a function that assigns to each state $s \in S$ a subset of actions from $A t$. This function provides us with convenience to find the actions that can be executed in certain states;

- $T_{t:}: A t \rightarrow 2^{S}$ is a function that assigns to each action $t \in A t$ a subset of states from $S$. This function provides us with convenience to find the states in which this action can be executed;

- $\tau:\left(S \times U_{n}\right) \rightarrow S$ is a state transition function that defines the state $\tau(s, \alpha)$ that would result by the performance of $\alpha$ from state $s ; U_{r}$ (defined afterwards) given here is the set of all possible sets of complex actions (also defined afterwards);

$-\pi: S \rightarrow 2^{p}$ is an interpretation function, which gives the set of primitive propositions satisfied in each state: if $p \in \pi(s)$, then proposition $p$ is true in state $\mathrm{s}$.

\subsection{Actions}

Actions play a key role in this unified model. When deciding 'who should achieve what in which way', the explicit representation of actions helps us to figure out how can agents obtain some state of affairs.

As shown in the unified model, $A t$ is the set of all actions. Bringing in the operators of propositional dynamic logic, a complex action can be defined as [15]: $\alpha::=t|\neg \alpha| \alpha \wedge \alpha|\alpha ; \alpha| \alpha$ ' $\alpha|\alpha *| \varphi$ ?, where the connectives have the usual interpretation, $t \in A t$, and $\varphi$ is a formula of NATEL which will be defined later. Removing all of the connectives within a complex action there will be atomic actions left, and we denote the set of all of these atomic actions $A_{\alpha}$. The set of all possible sets of complex actions is expressed as $U_{\alpha}$, and a set of complex actions is $u_{\alpha} \subseteq U_{\alpha}$. An action tuple for a coalition of agents $G$ is $\left\langle t_{l}, t_{2}, \ldots, t_{k}\right\rangle$, where $t_{i} \in A t_{i}$, for each $i \in G$. Action tuple is the same as joint action which has been defined in ATEL and NATL*. 


\subsection{Actions and Agents}

The ability of agents to cooperate to execute actions (what actions an agent or a coalition of agents can perform) and how this relates to their ability to reach certain states of affairs (the effects of actions) are worth investigated separately, but neither ATL nor its extended cooperation logics (ATEL, NATL*) has adopted such an approach [15]. As we know, the relation between actions and agents is complex: an action can be controlled by many different agents (that is, group action and common action), and an agent can control many different actions (that is, each agent $i \in A g$ is associated with a finite, non-empty set $A t_{i}$ of possible actions). Regarding this, several functions $\left(T_{c t}, T_{c t}, T_{t a}, T_{t c}, T_{s t}, T_{t s}\right)$ concerning the relation between agents and actions are given in detail to investigate the relationship in depth. When deciding 'who should achieve what in which way', these functions help us to figure out what actions an agent or a coalition of agents can perform, and help us to find out the agent or coalition of agents who is exactly controlling a single action.

It must be emphasized that by defining 'for each $i, j \in A g$, and $i \neq j$, it may be true that $A t_{i} \cap A t_{j} \neq \phi^{\prime}$ ', we can allow different agents to control the same action. This differs with the approaches taken by ATEL and NATL*.

\subsection{Actions and States}

Since the relation between actions and agents has been investigated in depth in last sub-section, we will research the relation between actions and states below.

As shown in the unified model, there are two functions. The first function, $T_{s t}: S \rightarrow 2^{A t}$ can help us to find the actions that can be executed in certain states. The second function, $T_{t s:}: A t \rightarrow 2^{S}$ can help us to find the states in which this action can be executed. Besides, these functions bring us convenience to define strategy and strategy tuple below [13], [14]. A strategy for an agent $i \in A g$ is a function: $\sigma_{i}: S \rightarrow A t_{i}$, which must satisfy the legality constraint that $\sigma_{i}(s) \in T_{s i}(s)$. A strategy tuple for a coalition $G=$ $\left\{a_{1}, \ldots, a_{k}\right\} \subseteq A g$ is $\sigma_{i}=\left\langle\sigma_{1}, \ldots, \sigma_{k}\right\rangle$, one for each agent $a_{i} \in G$. The set of all strategy tuples is denoted as $\Sigma_{G}$. An infinite sequence of states can be defined as $\lambda=\mathrm{s}_{0}, \mathrm{~s}_{1}, \ldots$; given $\mathbf{u} \in \mathbb{N}, \lambda[\mathrm{u}]$ is the component indexed by $\mathrm{u}$ in $\lambda$. The set of all infinite sequence of states is denoted as comp(s, $\left.\sigma_{G}\right)=$ $\left\{\lambda \mid \lambda[0]=\mathrm{s}\right.$ and $\left.\forall \mathrm{u} \in \mathbb{N}: \lambda[\mathrm{u}+1] \in\left\{\tau\left(\sigma_{i,}, \lambda[\mathrm{u}]\right)\right\}\right\}$. Strategy tuple is the same as strategy profile, and infinite sequence of states is the same as computation (run) which have been defined in ATEL and NATL*. As to the 
effects of actions (to bring about states of affairs), it can be expressed as $[\alpha] \varphi$. We will leave this to section 3 .

\subsection{Knowledge}

It is useful to bring knowledge into our framework since it helps us to do away with the strong and unrealistic assumption that agents know everything about the state of the system [13].

As shown in the unified model, $\sim$ is an epistemic accessibility relation for each agent $i \in A g$, and it represents indistinguishable states to agent $i$. The accessibility relations of a coalition of agents $G \subseteq A g$ is denoted by $\sim=$ $\left(U_{i \in G} \sim_{i}\right)$. The transitive closure of $\sim_{G}^{B}$ is denoted by $\sim_{G}^{C}$. The relation between actions and knowledge is bidirectional, since certain knowledge is required when agents want to execute actions properly, and after executing actions knowledge may be added to.

\subsection{Normative Ability}

The normative ability $\eta: A t \rightarrow 2^{S}$ is a function that defines a set of additional constraints on the actions that may be performed in any given state [14]. So that if $s \in \eta(t)$, then the normative ability $\eta$ forbids action $t$ from being performed when the system is in state $s$.

The relation between function $T_{t s}$ and function $\eta$ is worth investigated. Since function $T_{l s}$ defines whether or not an action can be executed in the context of the unified model, and function $\eta$ defines additional constraints on this actions, the requirement is that: $\forall t \in A t:\left(S \backslash T_{t s}(\mathrm{t})\right) \subseteq \eta(t)$. The operation of implementing the normative ability is thus an update on the unified model.

\section{NATEL}

Taking advantage of [13], [14], [15], the cooperation logic called the Normative Alternating-time Temporal Epistemic Logic (NATEL) is developed to extend ATL. It is the main contribution of us that actions, knowledge and normative ability can be represented in the object language at the same time other than only in the underlying semantics.

The syntax and semantics of NATEL are given as follows:

Definition 2. (THE SYNTAX OF NATEL)

The formal syntax of NATEL is given by the BNF grammar as:

$$
\varphi:==\text { true (truth constant) }
$$


$\mid p \quad$ (primitive propositions)
$\mid \neg \varphi \quad$ (negation)
$\mid \varphi \wedge \varphi \quad$ (conjunction)
$\mid[\eta: \alpha] \varphi \quad$ (effect of actions)
$\mid<<\eta: G \gg>\quad$ (what actions a coalition of agents can perform)
$\mid<<\eta: G>>0$ (in the next state, cooperative ability)
$\mid<<\eta: G>>\varphi$ (now and forever more, cooperative ability)
$\mid<<\eta: G>>\varphi u \varphi$ (until, cooperative ability)
$\mid K_{i} \varphi \quad$ (agent $i$ knows $\varphi$ )
$\mid E_{G} \varphi$ (everyone in coalition $G$ knows $\varphi$ )
$\mid C_{G} \varphi$ (it is common knowledge to everyone in the coalition $G$ that $\varphi$ ) In this definition, $p \in P$ is a propositional variable, $\alpha$ is a complex action, $\eta$ is a symbol denoting the normative ability, and $G \in U_{t i}$ is a set of agents.

As shown in the definition of syntax of NATEL, the syntax of ATL is extended by actions, knowledge and normative ability. For example, by $[\eta: \alpha] \varphi$, one can express the property of the effect of actions within the context of the normative ability $\eta$. Similarly, by $<<\eta: G>>0 \varphi$, one can express the property of what the coalition of agents $G$ can enforce to be true in the next state within the context of the normative ability $\eta$.

Definition 3. (THE SEMANTICS OF NATEL)

According to the unified model, the semantics of NATEL are given as follows:

$M, s \vDash$ true;

$M, s \vDash p$ iff $p \in \pi(s)$ (where $p \in P$ );

$M, s \vDash \neg \varphi$ iff $M, s \not \varphi$;

$M, s \vDash \varphi \wedge \psi$ iff $M, s \vDash \varphi$ and $M, s \vDash \psi$;

$M, s \vDash[\eta: \alpha] \varphi$ iff for all states $\tau(s, \alpha)$, it will be true that $M, \tau(s, \alpha)$

$\vDash \phi$ within the context of the normative ability $\eta$;

$M, s \vDash<<\eta: G>>\alpha$ iff for the set of all of the atomic actions of the complex action $\alpha$, it is true that $A_{\alpha} \subseteq\left(T_{c l}(G) \cap T_{s /}(s)\right)$ within the context of normative ability $\eta$;

$M, s \vDash<<\eta: G>>0$ iff $\exists \sigma_{i} \in \Sigma_{(i}$, such that $\forall \lambda \in \operatorname{comp}\left(s, \sigma_{i}\right)$, we have $M, \lambda[1]=\varphi$ within the context of the normative ability $\eta$;

$M, s \vDash<<\eta: G \gg>\square$ iff $\exists \sigma_{G i} \in \Sigma_{G i}$, such that $\forall \lambda \in \operatorname{comp}\left(s, \sigma_{G}\right)$, we have $M, \lambda[u] \models \varphi$ for all $u \in \mathbb{N}$ within the context of the normative ability $\eta$

$M, s \vDash<<\eta: G>>\varphi u \psi$ iff $\exists \sigma_{G i} \in \Sigma_{c_{i}}$, such that $\forall \lambda \in \operatorname{comp}\left(s, \sigma_{G}\right)$, there exist some $u \in \mathbb{N}$ such that $M, \lambda[u] \models \psi$, and for all $0 \leq v<u$, we have $M, \lambda[v] \vDash \varphi$ within the context of the normative ability $\eta$;

$M, s \vDash K_{i} \varphi$ iff for all $s^{\prime}$ such that $s \sim_{i} s^{\prime}: M, s^{\prime} \vDash \varphi$;

$M, s \vDash E_{G} \varphi$ iff for all $s^{\prime}$ such that $s \sim{ }_{G}^{E} s^{\prime}: M, s^{\prime} \vDash \varphi$; 
$M, s \vDash C_{G} \varphi$ iff for all $s^{\prime}$ such that $s \sim_{G}^{c} s^{\prime}: M, s^{\prime} \vDash \varphi$.

The other connectives (" $\vee$ ", " $\rightarrow$ ", " $\leftrightarrow$ ") can be defined by $\neg, \wedge$, and $<<\eta: G>>\diamond \varphi$ is shorthand for $\neg<<\eta: G>>\square \neg \varphi$.

Due to limit of space, a sound and complete axiom system for the cooperation logic (NATEL) is left to our next paper.

\section{A CASE STUDY}

Our example is a circuit model that consisting of some wire, a lamp (L), two switches $\left(\mathrm{K}_{1}, \mathrm{~K}_{2}\right)$, two electrical sources $\left(\mathrm{U}_{1}, \mathrm{U}_{2}\right)$, either switch $\mathrm{K}_{1}$ or switch $\mathrm{K}_{2}$ is turned on will make the lamp lighted. When both switches are turned on, the electrical current will pass directly through $\mathrm{K}_{1}$ and $\mathrm{K}_{2}$, and it leads to a clash. According to the circuit model and the unified model, the sets of states, agents, actions, atomic propositions are given as follows. $S=$ $\left\{s_{0}, s_{1}, s_{2}, s_{3}\right\}$, in which $s_{0}$ stands for $\mathrm{K}_{1}$-off and $\mathrm{K}_{2}$-off, $s_{1}$ stands for $\mathrm{K}_{1}$-off and $\mathrm{K}_{2}$-on, $s_{2}$ stands for $\mathrm{K}_{1}$-on and $\mathrm{K}_{2}$-off, $s_{3}$ stands for $\mathrm{K}_{1}$-on and $\mathrm{K}_{2}$-on; $A g$ $=\{1,2\} ; A t=\left\{1\right.$-on- $\mathrm{K}_{2}, 1$-off- $\mathrm{K}_{2}, 2$-on- $\mathrm{K}_{2}, 2$-off- $\mathrm{K}_{2}, 12$-on- $\mathrm{K}_{1}, 12$-off- $\left.\mathrm{K}_{1}\right\}$; $P=\left\{\mathrm{K}_{1}\right.$-off, $\mathrm{K}_{2}$-off, $\mathrm{K}_{1}$-on, $\mathrm{K}_{2}$-on $\} . \mathrm{K}_{1}$ can be turned on or turned off either by 1 or $2 ; \mathrm{K}_{2}$ can be turned on or turned off only by the cooperation of both 1 and 2 . The actions $1-$ on- $K_{2}$ and $1-$ off- $K_{2}$ can only be executed by agent $1,2-$ on- $\mathrm{K}_{2}$ and 2 -off- $\mathrm{K}_{2}$ can only be executed by agent 2,12 -on- $\mathrm{K}_{1}$ and 12-off- $\mathrm{K}_{1}$ are the actions that are allowed to be executed by different agents 1 and 2 . The relations between actions and agents, actions and states can be researched separately through functions involved actions. We give some examples of functions to show the relation between agents and actions here. $T_{a r}(1)=\left\{1\right.$-on- $\mathrm{K}_{2}, 1$-off- $\mathrm{K}_{2}, 12$-on- $\mathrm{K}_{1}, 12$-off- $\left.\mathrm{K}_{1}\right\}, T_{a r}(2)=\left\{2\right.$-on- $\mathrm{K}_{2}, 2$-off- $\mathrm{K}_{2}$, 12-on- $\mathrm{K}_{1}, 12$-off- $\left.\mathrm{K}_{1}\right\} ; T_{c l}(\{1,2\})=\left\{1\right.$-on- $\mathrm{K}_{2}, 1$-off- $\mathrm{K}_{2}, 2$-on- $\mathrm{K}_{2}, 2$-off- $\mathrm{K}_{2}, 12$ on- $\mathrm{K}_{1}, 12$-off- $\left.\mathrm{K}_{1}\right\} ; T_{t a}\left(1\right.$-on- $\left.\mathrm{K}_{2}\right)=1, T_{l d}\left(2\right.$-on- $\left.\mathrm{K}_{2}\right)=2 ; T_{l c}\left(12\right.$-on- $\left.\mathrm{K}_{1}\right)=\{1,2\}$. They bring us convenience to find the coalition of agents which is exactly controlling certain actions. Action $1-\mathrm{on}-\mathrm{K}_{2}$ is fully controlled by 1 , action $2-$ on- $\mathrm{K}_{2}$ is fully controlled by 2 , and action $12-\mathrm{on}-\mathrm{K}_{1}$ is only fully controlled by the coalition of 1 and 2 .

In order to avoid the situation of clash (that is, $s_{3}$ ), it is forbidden that both $\mathrm{K}_{1}$ and $\mathrm{K}_{2}$ are turned on at a given time. This additional constrain can be expressed by the follow normative ability.

$$
\eta(\alpha)=\left\{\begin{array}{l}
s_{0}, \text { if } \alpha=1 \text { on }-\mathrm{K}_{2} \wedge 2-\text { on }-\mathrm{K}_{2} \wedge 12-\text { on }-\mathrm{K}_{1} \\
s_{1}, \text { if } \alpha=12 \text {-on }-\mathrm{K}_{1} \\
s_{2}, \text { if } \alpha=1 \text {-on- } \mathrm{K}_{2} \wedge 2 \text {-on- } \mathrm{K}_{2}
\end{array}\right.
$$

This normative ability ensures that: when $\mathrm{K}_{1}$-off and $\mathrm{K}_{2}$-off, action 1-on- $K_{2} \wedge 2$-on- $K_{2} \wedge 12$-on- $K_{1}$ is forbidden to be executed; when $K_{1}$-off and $K_{2}-$ 
on, action 12-on- $\mathrm{K}_{1}$ is forbidden to be executed; when $\mathrm{K}_{2}$-off and $\mathrm{K}_{1}$-on, action 1 -on- $K_{2} \wedge 2-$ on $-K_{2}$ is forbidden to be executed.

Since NATEL takes actions, knowledge and normative ability into account at the same time, its expressive power and flexibility are much richer than the other two extended cooperation logics of ATL. It can express properties like: $M, s_{0} \vDash\left[\eta: 1-o n-\mathrm{K}_{2} \wedge 2\right.$-on- $\left.\mathrm{K}_{2}\right]\left(\mathrm{K}_{1}\right.$-off $\wedge \mathrm{K}_{2}$-on); its intended interpretation is that operating within the context of the normative ability $\eta$, executing action 1 -on- $\mathrm{K}_{2}$ and 2 -on- $\mathrm{K}_{2}$ at the same time in state $s_{\theta}$ can bring about state $s_{1}$. This shows the effect of actions; $M, s_{\theta} \vDash\left\langle<\eta: G>>O\left(\left(\mathrm{~K}_{1-}\right.\right.\right.$ off $\wedge \mathrm{K}_{2}$-on $) \vee\left(\mathrm{K}_{1}\right.$-on $\wedge \mathrm{K}_{2}$-off $)$ ); Its intended interpretation is that operating within the context of the normative ability $\eta$, in state $s_{0}$ the coalition of agent 1 and agent 2 has the ability to bring about state $s_{1}$ or $s_{2}$ (the next states of $\left.s_{\theta}\right)$. This shows the ability of coalitions.

In a word, we allow different agents to control the same actions and give functions that involved actions in more detail so that we can investigate the relations between actions and knowledge, actions and agents, actions and states separately. Furthermore, actions, knowledge and normative ability are represented in the object language other than only in the underlying semantics to improve the expressive power and flexibility of NATEL.

\section{CONCLUSIONS}

There are several efforts we have made in this paper. Firstly, different agents are allowed to control the same actions. It do away with the strong and unrealistic assumption of the other two extended cooperation logics of ATL (ATEL, NATL*), so our approach is better. Secondly, functions that involved actions are given in more detail, so that we can research the relations between actions and knowledge, actions and agents, actions and states in depth and separately. Thirdly, a unified model has been given. It combines the main components of [13], [14], [15], and at the same time includes components of our own. Lastly, a cooperation logic called NATEL has been developed as an extension to ATL. As a result, actions, knowledge and normative ability can be represented in the object language other than only in the underlying semantics. And since it takes actions, knowledge and normative ability into account at the same time, its expressive power and flexibility are much richer than the other two extended cooperation logics of ATL. In the 1990s, BDI (belief, desire, intention) was developed to represent the cognitive structure of agents [1]. We have done some work in this area too [2], [3], [4], [5]. It will be a wonderful attempt to combine our existing work in BDI and NATEL, and investigate the relations between them. 


\section{ACKNOWLEDGEMENTS}

This paper is supported by the National Natural Science Foundation of China under Grant No. 60373079, No. 60573076; and supported by the Foundation of the Chinese Academy of Sciences under Grant No. SYSKF0505.

\section{REFERENCES}

1. P. R. Cohen, H. I. Levesque.: Intention is choice with commitment, Artificial Intelligence, Vol 42 (1990) 213-261

2. Shanli Hu, Chunyi Shi.: The computational complexity of dynamic programming algorithm for combinatorial auctions. Proceedings of 2002 International Conference on Machine Learning and Cybernetics IEEE Vol 1 (2002.11) 266-268

3. Shanli Hu, Chunyi Shi and Xiuduan Fang.: The rationality of agents and the weak realism constraint for intentions. Proceedings of 2003 International Conference on Machine Learning and Cybernetics IEEE Vol 4 (2003.11) 2000-2003

4. Shanli Hu, Chunyi Shi.: Twin-subset semantic model for intention. Proceedings of 2003 International Conference on Machine Learning and Cybernetics IEEE Vol 1 (2003.11) 2004-2008

5. Shanli Hu, Chunyi Shi.: A dynamic model of multi-agent system. Proceedings of 2004 International Conference on Machine Learning and Cybernetics IEEE Vol 1 (2004.8) 183187

6. R. Alur, L. de Alfaro, T. A. Henzinger, S. C. Krishnan, F. Y. C. Mang, S. Qadeer, S. K. Rajamani, and S. Ta_siran.; MOCHA user manual. University of Berkeley Report, 2000

7. R. Alur, T. A. Henzinger, and O. Kupferman: Alternating-time temporal logic. Journal of the ACM, Vol 49, No. 5 (2002.9) 672-713

8. M. Pauly.: Logic for social software. PhD thesis, University of Amsterdam (2001)

9. M. Pauly.: A modal logic for coalitional power in games. Journal of Logic and Computation, Vol 12, No. 1 (2002) 149-166

10. W. van der Hoek, M. Wooldridge.: On the logic of cooperation and propositional control. Artificial Intelligence, Vol 64, No. 1-2 (2005) 81-119

11. W. van der Hoek M. Wooldridge: Model Checking Cooperation, Knowledge, and Time A Case Study. In Research in Economics, 57(3) (2003.9) 235-265

12. W. van der Hoek, M. Wooldridge.: Cooperation, Knowledge, and Time: Alternating-time Temporal Epistemic Logic and its Applications. In Studia Logica, $75(1)$ (2003.10) 125157

13. W. van der Hoek, M. Roberts, and M. Wooldridge: Knowledge and Social Laws. In Proceedings of the Fourth International loint Conference on Autonomous Agents and Multi-Agent Systems (AAMAS-05), Utrecht, the Netherlands (2005.7)

14. M. Wooldridge, W. van der Hoek. On obligations and normative ability: towards a logical analysis of the social contract. Journal of Applied Logic, Vol 3 (2005) 396-420,

15. L. Sauro, J. Gerbrandy, W. van der Hoek, and M. Wooldridge.: Reasoning about action and cooperation. To be presented on Proceedings of the Fifth International Joint Conference on Autonomous Agents and Multi-Agent Systems (AAMAS-06), Hakodate, Japan (2006.5) 